\title{
Heurísticos en la representación del conocimiento: Estrategias de clasificación de categorías difusas y bien definidas
}

\author{
JAVIER SÁINZ \\ Departamento de Psicología Básica: Procesos Cognitivos \\ Universidad Complutense de Madrid
}

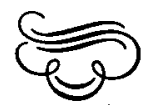

Resumen

La bipótesis que establece que el sistema ajusta sus descripciones a una transformación lineal de las propiedades que resultan del análisis perceptivo no está justificada y es evidente que la formación de categorias o conceptos es posible, aun tratando con categorias que no aceptan un modelo monotónico de representación. En esta investigación se analizan en dos experimentos las estrategias que permiten describir un cierto dominio de conocimiento en términos de un sistema binario de categorias difusas o bien definidas. Las descripciones que los sujetos efectúan suponen la formación de un sistema no monotónico de reglas de inferencia que permite distribuir los estimulos en clases complementarias, según contribuyan al proceso de decisión. Esta distribución no lineal de los estímulos en términos de las alternativas de respuesta es la que determina frente a los modelos de categorización propuestos hasta la fecha del proceso de categorización.

\section{Abstract}

The bypothesis that establishes that the system adjusts its descriptions to a linear transformation of the properties that result from the perceptual analysis has not been justified. These is evidence about the formation of categories or concepts even when these categories do not fit with a monotonic model of representation. Two experiments analyzed the strategies that allow for the description of a domain of knowledge in terms of a binary system of difuses or well defined categories. We can infer, from subjects descriptions, the formation of a non-monotonic system of rules of inference that allow for the distribution of stimuli in complementary classes according to their contribution to the decission-making process. As opposed to the current model of categorization, it is proposed that the non-linear distribution of the stimuli in terms of the response alternatives is determinant of the categorization process.

Dirección del autor: Javier Sáinz. Departamento de Psicología Básica: Procesos Cognitivos. Facultad de Psicología. Universidad Complutense de Madrid. Somosaguas. 28033 Madrid. 


\section{INTRODUCCION}

La naturaleza no monotónica de los procesos de pensamiento comienza a ser en la psicología contemporánea un tema habitual. El término de monotonicidad expresa que «una proposición debe derivarse de una serie de proposiciones, si se deriva de un conjunto menor de las que dicha serie contiene cuando se aceptan como premisas» (Sáinz y González-Marqués, 1986). En efecto, esta propiedad parece indudable en el razonamiento, se reconoce en los procesos heurísticos de resolución de problemas y se observa, en fin, y adquiere densidad, en el lenguaje afectando a la misma noción de verdad, a la noción que es lícita adoptar para referirse a lo que en lógica de predicados Aristóteles describía como el problema de la sinonimia esencial: la relación epistémica de los enunciados con la realidad que aprehenden.

El reconocimiento de este carácter no monotónico de los procesos de pensamiento no es, sin embargo, sino la punta del iceberg de un problema al que no se sustrae el más general de cómo se representa el conocimiento. Sin duda, aquél puede cuestionar algunas de nuestras más profundas convicciones, de nuestros más arraigados y sutiles hábitos de pensamiento: el realismo con que tratamos nuestras creencias y específicamente las que derivan de la percepción. En la medida en que la percepción es un proceso central, una justificación suficiente de este proceso requiere dar cuenta, con Fodor (1983) y Pylyshin (1984), de cómo le afecta esta propiedad, integrándola, al tiempo, incluso, en parte, frente a ellos, en una teoría funcional de la conducta. Hochberg (Peterson y Hochberg, 1983) se ha referido directamente a esta teoría desde una perspectiva metodológica —el método de conjunto opuesto-, una teoría que integra la percepción con los procesos centrales de formación y manipulación de creencias, lo que justifica su carácter constructivo.

El análisis perceptivo se ejecuta, a través de canales ajustados, a distintas frecuencias espaciales, con independencia de su relevancia, pero su registro (cf. Marcel, 1983a, b) depende de los esquemas activos en el procesador, de la satisfacción de demandas de distinta naturaleza (Farell, 1984; Foard y Kemler Nelson, 1984, y Nosofski, 1985), a saber las que vienen definidas por la naturaleza simbólica de la conjunción de los resultados de este proceso de análisis, es decir, de aquello que decide su identificación como objeto, de su naturaleza significativa como miembro de una clase de acontecimientos (cf. Newell, 1982). Hay en esto dos problemas: un problema es la descripción física del estímulo, es decir, qué clase de sucesos físicos son sintácticamente relevantes como componentes de una representación; otro problema es cómo una cierta serie de sucesos físicos se tratan como una invariante o una regularidad relevante, como una descripción, un dato o clase de acontecimientos. Los modelos de categorización se proponen precisamente para definir qué descripciones resultan ser básicas o primitivas para el sistema, cómo se han establecido, cómo se usan o se manipulan y transforman, y en términos de qué condiciones a nivel sintáctico-simbólico y a nivel semántico (Sảinz y González-Marqués, 1986). Qué duda cabe que el rendimiento de tales descripciones, de las categorías en que se describe la realidad, depende de en qué medida estas descripciones preserven la información potencial de los acontecimientos, más aún, de en qué medida preserven las relaciones explícitas en éstos (cf. Mervis y Rosch, 1981). De 
acuerdo con este planteamiento parecería que los modelos de categorización sólo pueden ser lineales, es decir, sólo pueden atenerse a una descripción que se agota en la definición de relaciones de contingencia entre sucesos físicos discriminables en los estímulos (cf. Fried y Holyoak, 1984; Knapp y Anderson, 1984; Richardson y Bhavnani, 1984, y Smith y Medin, 1981). Sin embargo, ni siquiera este tipo de información preserva mejor la naturaleza de la que contiene el estímulo desde la perspectiva del sistema que se procesa. Una definición convencional del proceso de categorización subraya, al mismo tiempo que la especificidad de los acontecimientos, la necesidad que presenta el sistema de integrarlos en clases de identidad y de equivalencia.

Una clase de equivalencia, en efecto, no se ajusta por definición a un modelo lineal o monotónico de categorización. Hinton (1981) ha proporcionado algunos ejemplos de estímulos que los modelos lineales no pueden asociar de forma apropiada y Minsky y Papert (1969) han demostrado la incapacidad de estos modelos para derivar una figura geométrica conexa a partir de una figura geométrica cuyos lados se presentan inconexos. La naturaleza no monotónica de los procesos que afectan a la categorización de un estímulo ha sido incluso reconocida a nivel del propio sistema nervioso y hay una buena muestra de fenómenos que la avalan (Anderson, Silverstein, Ritz y Jones, 1977; Anderson y Silverstein, 1978). Parece evidente -por ejemplo, en el habla - que la variación continua de las dimensiones de estímulo no implica que el cambio psicológico sea también lineal (Massaro y Hary, 1984). La hipótesis que establece, en fin, que el sistema ajusta sus descripciones a una transformación lineal de las propiedades que resultan del análisis perceptivo no está justificada y es evidente que la formación de categorías o conceptos es posible aun tratando con categorías que no aceptan un modelo monotónico de representación (Fried y Holyoak, 1984; Knapp y Anderson, 1984; Neumann, 1977; Oden, 1977;; Osherson y Smith, 1982; Roth y Mervis, 1983, y Smith y Osherson, 1984). Por eso han fracasado los modelos que pretenden medir la complejidad del estímulo y derivar su interpretación a partir de su mera descripción (cf. Buffart, Leeuwenberg y Restle, 1981; 1983). Al tiempo que se desarrollan lógicas no monotónicas que permiten estudiar el razonamiento humano (McDermott y Doyle, 1980; McDermott, 1982, y Moore, 1985) y se estudian las estrategias de clasificación en la solución de problemas complejos de categorización en tanto determinan cómo el sistema cognitivo reconoce su medio, dado que también en su descripción actúan procesos de inferencia de la misma clase general.

Los modelos de categorización que se han propuesto o bien suponen la naturaleza formal del núcleo conceptual o suponen que la naturaleza del núcleo se ajusta a una distribución estocástica de propiedades. En tanto el primer tipo de modelos defiende el carácter reglado del núcleo conceptual, el segundo define la estructura interna del núcleo recurriendo al concepto de prototipo. Un prototipo es una representación sumaria de la distribución de los miembros de una categoría (Smith y Medin, 1981). Los miembros de un concepto se definen, según esta hipótesis, por su relación con el prototipo de la categoría a la que pertenecen. Sin embargo, estas hipótesis, en rigor, identifican el proceso de categorización con un proceso lineal o monotónico según el cual la asignación de una categoría deriva de 
las propiedades que es posible reconocer en una serie de estímulos y de relaciones que se establecen sólo por referencia a estas propiedades físicas. A pesar de esta nota común, estas hipótesis no resuelven del mismo modo la naturaleza de este proceso de categorización ni pueden adaptarse de la misma manera, si se modifican en este sentido, a la hipótesis general que defiende el carácter no monotónico de sus descripciones.

En relación con este carácter, una descripción es: a) exhaustiva o completa, lo que supone el reconocimiento inequívoco de un estímulo cualquiera sea el contexto en que se presente - alternativa que se asocia a un isomorfismo en sentido fuerte-; o b) restrictiva o autofinalizada, lo que supone que un estimulo se identifica por restricción de incertidumbre. Existen, por tanto, teóricamente otras alternativas de clasificación distintas de la que lo determina en un contexto o en un dominio implícito de referencia.

El modelo clásico supone la naturaleza formal del núcleo conceptual. El núcleo conceptual responde a una regla lógica que vincula rasgos o dimensiones discriminables en el estímulo. Si se confunde la identificación de un estímulo con su representación conceptual —un corolario de la hipótesis que asegura el carácter exhaustivo o completo de una descripción-, esta hipótesis de la naturaleza formal del núcleo no permite explicar algunos de los hechos mejor conocidos del proceso de categorización, a saber, la indefinición de los límites de una categoría (Andersen, 1975; Labov, 1973; Lakoff, 1973; Osherson y Smith, 1982; Roth y Mervis, 1983); la indefinición de la relación de membrecía - a menudo confundida con el fenómeno de tipicidad- (Amstrong, Gleitman y Gleitman, 1983), y la indefinición de las relaciones jerárquicas entre los conceptos (Hampton, 1982; Markman, 1984; Osherson y Smith, 1981).

El modelo clásico, sin embargo, no identifica la representación de un concepto y el proceso por el que se asigna, no afirma que exista un número fijo de rasgos idénticos y, no obstante, específicos de todos y cada uno de los ejemplares que se consideran miembros. Parece haber en esto una confusión (cf. Smith y Medin, 1981) entre los postulados teóricos del modelo clásico y su traducción operacional en el laboratorio. La hipótesis crítica es la naturaleza formal del núcleo conceptual, independientemente de qué lógica permita caracterizarla. La distinción entre categorías difusas y bien definidas es falaz en cuanto se trata con un sistema no monotónico de reglas de inferencia que determinan a qué respuesta se asignan las distintas alternativas de estímulo en un dominio (Farell, 1984; Sáinz, 1985a, 1985b, 1986). Definir este sistema de reglas es tanto más difícil por cuanto un proceso de decisión no compromete necesariamente todas y cada una de las propiedades estructurales que definen el núcleo conceptual de una categoría (Chaffin, 1981).

Las anomalías teóricas y empíricas del modelo clásico - resultado de interpretar el modelo como si defendiera que la asignación de una categoría exige identificar una serie de rasgos y de relaciones lógicas idénticas en todos y cada uno de sus miembros potenciales- permitieron que surgiera como alternativa la hipótesis distribucional. Los modelos probabilísticos y de ejemplar difieren, en efecto, del modelo clásico en la estructura que atribuyen al núcleo de una categoría, de carácter distribucional. Esta concepción se expresa en términos de un prototipo cuyos rasgos o dimensiones reflejan la variabilidad inferida de los miembros que diagnostica (Hamp- 
ton, 1979; McCloskey y Glucksberg, 1979; Rosch y Vervis, 1975). Un concepto se asigna si posee un número crítico o una suma ponderada de propiedades de las que el prototipo de la categoría reconoce (Smith y Medin, 1981). Los modelos de ejemplar adoptan como representación sumaria de un concepto un cierto subconjunto de ejemplares miembros más que un inventario de propiedades significativas y abstractas (Brooks, 1978; Medin y Schaffer, 1978; Mervis, 1980; Reber y Allen, 1978; Reed y Friedman, 1973; Rosch, 1978; Smith y Medin, 1981). Cómo se establece el ejemplar o ejemplares que sirven como términos comparativos varía de acuerdo con las especificaciones de cada modelo. Dado que los modelos de ejemplar adoptan como representación sumaria un cierto conjunto de ejemplares, miembros del concepto, y que la representación progresa abstrayendo las dimensiones o rasgos críticos que justifican la comparación (cf. Medin y Schaffer, 1978), los modelos de ejemplar no difieren formalmente de los modelos probabilísticos (Sáinz, 1985a).

El modelo de semejanza familiar supone que la estructura interna de una categoría responde a alguna distribución de rasgos que puede determinarse a partir de sus miembros (Rosch y Mervis, 1975). El modelo de prototipo puede definirse como el resultado de calcular el centroide de una categoría a partir de las coordenadas de cada ejemplar en un espacio euclidiano común definido por sus dimensiones (Aiken, Fenker y Evans, 1974; Smith y Medin, 1981). El modelo de densidad categorial (Fried y Holyoak, 1984) es idéntico al modelo de prototipo excepto en el hecho de que este último no permite registrar la variabilidad de los ejemplares respecto al prototipo que los representa. El modelo de densidad categorial supone que la clasificación de los ejemplares está en función de las distribuciones de densidad de las respectivas categorías. Esta función se expresa de acuerdo con el teorema de Bayés, calculando la razón proporcional que existe entre la probabilidad asociada de un ejemplar y de la categoría a la que pertenece y las probabilidades asociadas de los ejemplares y las categorías alternativas a las que pueden pertenecer. Esta función bayesiana se modifica de acuerdo con un proceso cíclico de revisión de parámetros que modifica la distribución de la categoría sobre un espacio de rasgos.

El modelo de contexto (Medin y Schaffer, 1978) supone que la clasificación de un ejemplar implica, de un modo u otro, una configuración o combinación de propiedades (Medin, 1983; Malt y Smith, 1984). La semejanza de dos ejemplares en una categoría se define de acuerdo con una regla multiplicativa a partir de los rasgos con que se identifican y/o difieren. De acuerdo con esta regla, el número de ejemplares a que un cierto ejemplar es similar es más importante a la hora de su clasificación que su semejanza media a los miembros de la categoría a que pertenece. El modelo de contexto difiere del modelo de prototipo en la regla de identificación que propone: la clasificación de un ejemplar es directamente proporcional a su semejanza respecto de los que son miemhros de una categoría e inversamente proporcional a su semejanza respecto de todos los ejemplares del contexto. Los parámetros de semejanza del modelo de contexto se determinan empíricamente a partir de la clasificación de los distintos ejemplares por un algoritmo de regresión no lineal que trata de maximizar el número de clasificaciones correctas que se obtienen (Medin, Altom y Murphy, 1984, y Medin y Schaffer, 1978). 
Estos modelos no sirven sino para describir, en parte, qué clase de propiedades o rasgos no son sintácticamente relevantes como componentes de una representación. La mayor parte de los problemas derivan de cómo se justifica la hipótesis de monotonicidad de la distribución de las categorías y de las propiedades en que éstas se reconocen. Esta hipótesis se enfrenta al hecho de que «sólo los ejemplares de las categorías proporcionan una representación acerca de la distribución de las categorías" (Fried y Holyoak, 1984 , p. 255) y, sin embargo, ningún modelo supone que se codifican específicamente todos y cada uno de los ejemplares. Excepto si se acepta a priori esta representación, estos modelos desconocen cómo variar la distribución si ésta es no monotónica. La hipótesis de monotonicidad no se justifica y no puede, de hecho, imponerse con carácter general (cf. Flannagan, Fried y Holyoak, 1981; Neumann, 1977, y Roth y Mervis, 1983). Es claro, en este contexto, que los distintos modelos ni aportan solución alguna a las que creen anomalías del modelo clásico, ni suponen una justificación de cómo procede el sistema cognitivo cuando reconoce y describe su medio.

En todos estos modelos, el proceso de categorización se interpreta como la identificación terminal de los ejemplares que se someten a clasificación en una tarea experimental. De hecho, formalmente no difieren en los supuestos en que se apoyan. Los módelos probabilísticos y los modelos de ejemplar no distinguen entre identificación y categorización del estímulo, reduciéndose a proporcionar una descripción de los rasgos que son relevantes en una tarea que exige la comparación de los estímulos que se presentan. Estos procesos de identificación y categorización pueden distinguirse entre sí sólo si se definen las condiciones en que se procesa la información, y si los miembros de un concepto natural se definen con independencia de sus descripciones estructurales. Cuando la identificacion y la categorización suponen el mismo tratamiento del estímulo, su descripción paramétrica permite predecir cómo se comporta el sistema cuando procede a su clasificación. En otros términos, el reconocimiento o identificación de un estímulo es equivalente a su clasificación cuando este proceso no requiere una transformación no monotónica de la información que contiene. Esta transformación viene exigida por la tarea. Los modelos de categorización propuestos hasta la fecha tienden a identificar la clasificación del estímulo con su identificación tal como se deduce de los procedimientos de identificación que aplican (Sáinz, 1985a).

Nosofski (1984) ha demostrado que la regla del modelo de contexto es formalmente idéntica a la regla de respuesta del modelo de elección de Luce (1963), ya que se deriva de los mismos supuestos en relación con la identificación del estímulo. Ambas reglas se caracterizan por defender una hipótesis de correspondencia según la cual las relaciones biyectivas contingentes entre estímulos y respuestas en la identificación se transforman en la clasificación en relaciones sobreyectivas contingentes entre los mismos estímulos y respuestas. Puede predecirse la ejecución en la clasificación a partir de la identificación simplemente haciendo corresponder ambas matrices, sabiendo que lo único que varían son las alternativas de respuesta. Nosofsky (1984) interpreta la regla de procedimiento del modelo de contexto de acuerdo con los desarrollos teóricos de los métodos de escalamiento multidimensional: «la regla que calcula la semejanza del estímulo surge como un caso especial de la distancia psicológica entre los estímulos, que 
se conforma a una métrica de bloque, y de la semejanza de estímulo, que es una función decreciente exponencial de distancia psicológica (...) el modelo de contexto de Medin y Schaffer (1978) se deriva de integrar la hipótesis de correspondencia de la relación entre la identificación y la clasificación del estímulo con (...) modelos bien definidos en el ámbito de la elección y de la semejanza» (Nosofsky, 1984, p. 107).

El análisis de Nosofsky (1984) permite concluir que el modelo de contexto no distingue formalmente entre identificación y categorización. Estos procesos sólo difieren si las demandas relativas y contingentes de cada tarea son también distintas. Tampoco los modelos que se han propuesto difieren en este aspecto del modelo de contexto, ya que, de hecho, sólo difieren entre sí no en cómo caracterizan el núcleo conceptual, sino en qué procedimientos permiten identificar sus ejemplares.

El modelo de densidad categorial no difiere esencialmente del modelo de contexto, ya que puede tratarse como un caso límite si se supone que la regla de relativa verosimilitud es formalmente idéntica a la regla multiplicativa de Medin y Schaffer (1978). Basta suponer que cada instancia o ejemplar determina una distribución equivalente a un gradiente de generalización alrededor de un punto en el espacio de rasgos que viene definido por el ejemplar. La regla de relativa verosimilitud predice entonces que la probabilidad subjetiva de un cierto ejemplar dada una categoría será proporcional a la suma de las probabilidades subjetivas del ejemplar, dadas las distribuciones asociadas con cada uno de los ejemplares registrados en dicha categoría, suponiendo que sean igualmente comunes (Fried y Holyoak, 1984). El modelo de densidad categorial trata con las distribuciones relativas a las categorías, pero estas distribuciones se infieren de los ejemplares en función de los que se estiman los parámetros del modelo. Una vez que se ha aceptado la identidad de ambos modelos, el modelo de densidad categorial se deriva de las distribuciones de los respectivos ejemplares de una categoría y el modelo de contexto es meramente convencional, a saber, la que se deduce de tratar con diferentes niveles categoriales.

El modelo de prototipo no difiere, por su parte, del modelo de densidad categorial excepto en que la regla de relativa verosimilitud no se comporta como una regla aditiva de información dimensional independiente sino que, aun conservando la independencia estadística de las dimensiones, la regla es una combinación multiplicativa de información. Ya Pitz y Leung (1977) relacionaron el modelo de prototipo con un enfoque estadístico de la decisión. Ambos modelos difieren exclusivamente en que el modelo de prototipo no tiene en cuenta la variabilidad de las categorías, aunque ésta no es una propiedad que afecte a sus hipótesis básicas.

Si modificamos el modelo de prototipo en términos de un modelo de indicadores relacionales o interactivos, podemos efectuar predicciones, incluso más ajustadas que el modelo de contexto, de los resultados que se obtienen en una prueba de clasificación. La única diferencia que existe entre el modelo de prototipo y el modelo de contexto reside en la regla que define cómo se reconocen los miembros de una categoría. En el modelo de prototipo se considera la semejanza de cada ejemplar al prototipo de su categoría, en tanto que en el modelo de contexto se considera la semejanza de cada ejemplar a los que se presentan. Depende, sin embargo, de qué tipo de descripción se adopte para que un prototipo contenga información acer- 
ca de la distribución, en cuyo caso estas diferencias no son ya aparentes.

En suma, los modelos de prototipo y densidad categorial y el modelo de contexto no difieren respecto a cómo debe caracterizarse una categoría sino sólo en las reglas que proponen y aceptando las condiciones de procedimiento son expresión de los mismos supuestos básicos, supuestos que tienden a identificar la descripción estructural de un estímulo con su clasificación. De acuerdo con estas observaciones, parece razonable distinguir entre identificación y categorización y reconocer a partir de esta distinción la adecuación descriptiva y explicativa de cada modelo.

En primer término, trataremos de reconocer si estos modelos permiten definir el proceso de categorización o si este proceso puede, por el contrario, caracterizarse en términos de una regla de carácter formal independientemente de la distribución de los ejemplares y de las categorías en que se agrupan. En segundo término, trataremos de reconocer si las descripciones que se derivan de la clasificación de los ejemplares en una serie de categorias derivan de una transformación lineal de la información del estimulo o, por el contrario, derivan de una transformación no monotónica de sus propiedades, tal y como éstas se reconocen en su descripción estructural. Nuestra tesis es que estos modelos de categorización al confundir los procesos de identificación y categorización sólo son apropiadas en la medida en que permiten describir las propiedades de los estimulos que se someten a clasificación. Por tanto, sólo en la medida en que su clasificación exija procesar la misma información que para su reconocimiento, resultan ser adecuados. Si esto es así, el ajuste de los distintos modelos nos permitirá reconocer la actuación de los procesos que intervienen en la categorización, que suponemos son de naturaleza no monotónica.

\section{EXPERIMENTOS 1A-1B}

Dado que el proceso de categorización depende del análisis perceptivo, es preciso proveernos de una descripción de las alternativas de estímulo suficientemente adecuada a nuestros propósitos. Los métodos de escalamiento multidimensional nos proporcionan esta descripción. Sin embargo, y a pesar del carácter separable de los rasgos relevantes de los estímulos que se presentan (cf. fig. 1), el análisis de escalamiento que se lleva a cabo no se atiene a una métrica de bloque. La tesis de que cada clase de estímulo se ajusta a un tipo específico de métrica ha sido puesta en cuestión por Dunn (1983) y Nosofsky (1985), por lo que no parece razonable mantenerla en este caso. Siguiendo una métrica euclidiana es posible representar en un espacio común las semejanzas y diferencias que los sujetos experimentales reconocen en nuestros estímulos.

.En el primer experimento de esta serie (Exp. 1A) los estímulos pueden clasificarse en dos categorías, según rasgos que comparten todos y cada uno de sus respectivos miembros. Las categorias en este experimento se definen, en efecto, en términos de una regla conjuntiva que especifica la variación conjunta de dos rasgos que resultan ser críticos para identificar los distintos ejemplares. En la tabla I se presenta su distribución. En el segundo experimento de esta misma serie (Exp. 1B), los estímulos se clasifican de forma más compleja: un ejemplar es miembro de una categoría cuando se 
TABLA 1

Estructura de las categorias bien definidas (Exp. 1A)

\begin{tabular}{|c|c|c|c|c|c|c|c|c|c|c|c|c|c|c|c|c|c|c|c|}
\hline Cat & 1 & 2 & 3 & 4 & 5 & 6 & $\mathbf{R}$ & & sos & Cat & 1 & 2 & 3 & & 5 & 6 & $\mathbf{R}$ & & sos \\
\hline A01 & 1 & 1 & 1 & 1 & 1 & 1 & 4 & 52 & 608 & B01 & 1 & 1 & 2 & 2 & 2 & 1 & 1 & 46 & 388 \\
\hline & 1 & 1 & 1 & 1 & 1 & 2 & 3 & 51 & & ? & $i$ & 1 & 2 & 2 & 2 & 2 & 2 & 47 & 412 \\
\hline A03 & 1 & 1 & 1 & 1 & 2 & 1 & 3 & 48 & 572 & B03 & 2 & 1 & 2 & 2 & 2 & 1 & 2 & 47 & 412 \\
\hline A & 1 & 1 & $i$ & 1 & 2 & 2 & 2 & 47 & 54 & & 2 & 1 & 2 & 2 & 2 & 2 & 3 & 48 & 436 \\
\hline A & 1 & 1 & 2 & 1 & 1 & 1 & 3 & 48 & 568 & B05 & 1 & 2 & 2 & 1 & 1 & 1 & 1 & 46 & 428 \\
\hline $\mathrm{AO}$ & 1 & 1 & 2 & 1 & 1 & 2 & 2 & 47 & 544 & B06 & 1 & 2 & 2 & 1 & 2 & 2 & 2 & 47 & 542 \\
\hline $\mathrm{AC}$ & 2 & $i$ & 1 & 1 & 1 & 1 & 3 & 51 & 58 & & 1 & 2 & 2 & 2 & 2 & 1 & 2 & 50 & 484 \\
\hline A & 2 & 1 & 1 & 1 & 1 & 2 & 2 & 50 & 560 & B08 & 1 & 2 & 2 & 2 & 2 & 2 & 3 & 51 & 508 \\
\hline $\mathrm{AO}$ & 2 & 1 & 1 & 1 & 2 & 1 & 2 & 47 & 548 & B09 & 2 & 2 & 2 & 1 & 2 & 1 & 2 & 47 & 452 \\
\hline A1 & 2 & 1 & 1 & $i$ & 2 & 2 & 1 & 46 & 57 & B10 & 2 & 2 & 2 & 1 & 2 & 2 & 3 & 48 & 476 \\
\hline & 2 & 1 & 2 & $i$ & 1 & 1 & 2 & 47 & 54 & B 11 & 2 & 2 & 2 & 2 & 2 & 1 & 3 & 51 & 508 \\
\hline A12 & 2 & 1 & 2 & 1 & 1 & 2 & 1 & 46 & 520 & B12 & 2 & 2 & 2 & 2 & 2 & 2 & 4 & 52 & 532 \\
\hline
\end{tabular}

asemeja más a los miembros de su categoría que a los de la categoría alternativa. La semejanza de un ejemplar se calcula sumando el número de veces que se presenta una cierta propiedad en los miembros dè su categoría y sumando las sumas parciales que se corresponden con todas y cada una de las propiedades del mismo ejemplar. En la tabla II se presenta su distri-

TABLA II

Estructura de las categorias de estructura difusa (Exp. 1B)

\begin{tabular}{|c|c|c|c|c|c|c|c|c|c|c|c|c|c|c|c|c|c|}
\hline Cat & 1 & 2 & 3 & 4 & 5 & 6 & Propia & Altva & Cat & 1 & 2 & 3 & 4 & 5 & 6 & Propia & Altva \\
\hline A01 & 2 & 1 & 1 & 1 & 1 & 1 & 41 & 31 & B01 & 1 & 2 & 2 & 1 & 1 & 1 & 35 & 37 \\
\hline $\mathrm{AO}$ & 2 & 1 & 2 & 2 & 1 & 1 & 43 & 29 & B02 & 1 & 1 & 1 & 2 & 2 & 1 & 41 & 31 \\
\hline $\mathrm{AO} 3$ & 2 & 2 & 1 & 1 & 2 & 1 & 41 & 31 & $\mathrm{~B} 03$ & 1 & 1 & 2 & 1 & 1 & 1 & 39 & 33 \\
\hline A04 & 1 & 1 & 1 & 2 & 1 & 1 & 35 & 37 & B04 & 1 & 1 & 2 & 1 & 1 & 2 & 43 & 29 \\
\hline A05 & 2 & 2 & 1 & 2 & $i$ & 1 & 47 & 25 & B05 & 2 & 1 & 1 & 1 & 2 & 2 & 39 & 33 \\
\hline A06 & 1 & 2 & 1 & 1 & 1 & 1 & 37 & 35 & B06 & 1 & 1 & 1 & 1 & 2 & 2 & 47 & 25 \\
\hline A07 & 2 & 2 & 2 & 1 & 2 & 2 & 37 & 35 & B07 & 1 & 2 & 2 & 1 & 2 & 2 & 43 & 29 \\
\hline A08 & 2 & 2 & 2 & 1 & 2 & 1 & 41 & 31 & B08 & 1 & 2 & 1 & 1 & 2 & 2 & 43 & 29 \\
\hline A09 & 2 & 2 & 2 & 2 & 1 & 2 & 43 & 29 & B09 & 1 & 2 & 2 & 2 & 1 & 2 & 39 & 33 \\
\hline A 10 & 2 & 1 & 2 & 2 & 1 & 2 & 39 & 33 & B10. & 2 & 1 & 1 & 2 & 2 & 2 & 39 & 33 \\
\hline A11 & 2 & 2 & 1 & 2 & 1 & 2 & 43 & 20 & B11 & 1 & 1 & 1 & 2 & 2 & 2 & 45 & 27 \\
\hline A12 & 2 & 2 & 2 & 2 & 2 & 1 & 43 & 29 & B12 & 1 & 1 & 2 & 2 & 2 & 1 & 41 & 31 \\
\hline
\end{tabular}

bución. En tanto, el núcleo conceptual de las categorías del primer experimento presenta una estructura bien definida, la estructura interna de las categorías del segundo experimento es difusa. Estos experimentos tienen por objeto proporcionar una descripción estructural de los estímulos que permita predecir cómo ha de resultar su clasificación.

\section{Método}

\section{Sujetos}

En este experimento participaron 26 sujetos, estudiantes universitarios, que se prestaron voluntariamente a colaborar en esta investigación. Trece 
de ellos en el experimento $1 \mathrm{~A}$ que empleaba categorías bien definidas y otros 13 en el experimento $1 \mathrm{~B}$ que utilizaba, por el contrario, categorías de estructura difusa.

\section{Procedimiento}

Los estímulos gráficos que se utilizaron consisten en dibujos geométricos que varían en seis rasgos (cf. fig. 1). En ambos experimentos los estímulos pueden clasificarse en dos categorías, A y B, 12 en cada categoría, 24 en total. La estructura interna de estas categorías se recoge en las tablas I

\section{FIGURA 1}
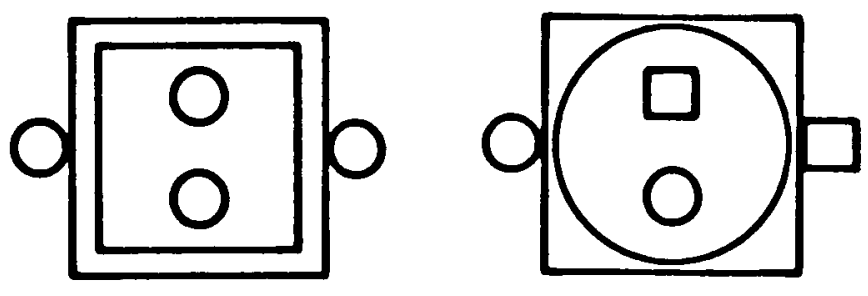

Par ejemplar.

y II. En estas tablas, 1 representa un cuadrado y 2 un círculo. Las columnas representan, por este orden, las figuras externa, inscrita, superior interna, inferior interna, figura del margen izquierdo y figura del margen derecho.

En cada uno de estos experimentos se formaron todas las combinaciones de pares posibles en los dibujos respectivos, sin que se repitiera el par o se presentara un par de dibujos idénticos. En total se obtuvieron 276 pares en cada uno de ellos. Estos pares se presentaron en un orden casual a través de dos proyectores Kodak Carousel 2050AV controlados por un computador Hewlett Packard (HP 9825A). Los estímulos permanecían a la vista del sujeto durante siete segundos. La tarea de los sujetos consistía en juzgar la semejanza global del par sobre una escala de 1 (un par muy semejante) a 9 (un par muy diferente). Los sujetos ofrecían su respuesta ante un micrófono, lo que permitía a un auxiliar de investigación registrarla en el computador. Estas experiencias eran por completo independientes, durando cada una de ellas cincuenta minutos aproximadamente por la búsqueda aleatoria del par a presentar.

\section{Resultados y discusión}

Las matrices de puntuaciones obtenidas en cada uno de estos experimentos se sometieron al método Indscal de escalamiento multidimensional (Kruskal y Wish, 1978; Falmagne, 1985), según el paquete de programas MSX que a este efecto se encuentra disponible en la biblioteca de programas del Centro de Cálculo de la Universidad Complutense. El método Indscal proporciona un análisis de una matriz de datos tridimensional de medidas de semejanza por medio de un modelo de distancia ponderada que 
utiliza una transformación lineal de los datos. Este análisis tridimensional es un caso especial de la descomposición canónica de una serie de matrices de datos de n-dimensiones por un modelo de productos escalares. El método Indscal añade a esta representación especial otra donde sitúa a los sujetos, lo que permite diferenciar las estrategias de los sujetos al representarlos en un espacio específico. Este procedimiento no utiliza las distancias entre los puntos de espacio de estímulo. En su lugar forma una serie de configuraciones a partir de las puntuaciones proporcionadas por cada uno de los sujetos, sirviéndose después de las distancias entre estas configuraciones. Una configuración individual se forma alterando la configuración del espacio de estímulo de acuerdo con la matriz de pesos que se corresponde con las puntuaciones de cada sujeto. Aunque esto equivale a considerar que las puntuaciones individuales no son casuales, lo que necesariamente repercute en la representación espacial de los estímulos, esta información permite efectuar predicciones individuales en aquellos modelos de categorización que contemplan esta posibilidad. Una vez determinemos la distancia psicológica de los items presentados pueden calcularse las predicciones de los distintos modelos de categorización según sus reglas específicas.

\section{Experimento 1A. Catetorias de estructura bien definida}

Una solución de seis dimensiones puede ser suficiente para explicar la mayor parte de la varianza, aunque todavía es importante la que falta por explicar si se escoge esta solución. La configuración de puntos en el espacio de estímulo pone de manifiesto que los distintos estímulos se someten a análisis según los rasgos de que se constituyen. El sujeto parece discriminar la información sucesivamente según una serie de criterios que forma a medida que reconoce el conjunto de los estímulos que se le presenta (cf. fig. 2).

FIGURA 2

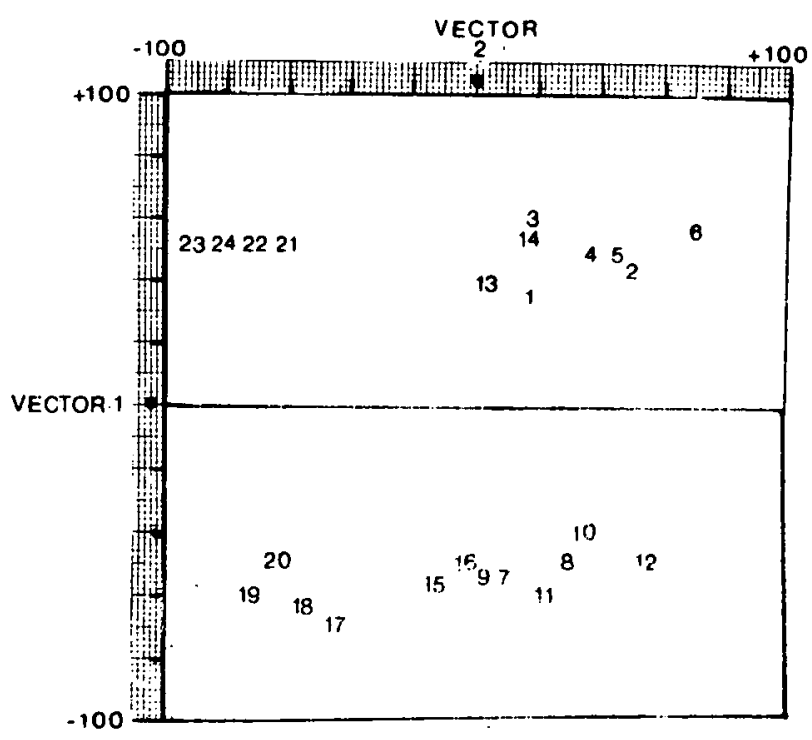

Representación de los estimulos. Exp. 1A. Categorias de estructuras bien definidas. 
Representación de los estímulos. Experimento 1A. Categorías bien definidas. La tabla III A, recoge la varianza que se explica en una solución de seis dimensiones. Podemos reconocer las distintas dimensiones analizando las puntuaciones de cada uno de los estímulos en la matriz de la configuración final. La primera dimensión es una propiedad configural: expresa la predominancia de cuadrados o de círculos entre las distintas alternativas de estímulo. Esta propiedad se identifica en el modelo de contexto por el parámetro G. Los parámetros $\mathrm{B}, \mathrm{A}, \mathrm{D}, \mathrm{C}$ y $\mathrm{E}$ expresan por este orden los siguientes rasgos: figura del margen izquierdo, externa, inferior interna, superior interna y figura del margen derecho. Escogiendo esta solución no se reconoce la influencia de la figura inscrita de tal modo que sólo los rasgos reseñados parecen explicar globalmente cómo los sujetos juzgan la semejanza de los pares de estímulo presentados. No obstante, y como se deduce de las correlacciones entre las puntuaciones que se obtienen en esta solución y los datos originales, los sujetos difieren en sus estrategias de descripción, confirmando que no identifican del mismo modo este dominio de estímulos (cf. tabla IVA).

TABLA III

Varianza explicada en una solución MDS para los experimentos $1 A$. Categorias bien definidas y $1 B$. Categorias de estructura difusa.

\begin{tabular}{cccc}
\hline Dim & Var. Acum. & Dim. & Var. Acum. \\
\hline 1 & 0.241374 & 1 & 0.353250 \\
2 & 0.425741 & 2 & 0.498872 \\
3 & 0.567223 & 3 & 0.609045 \\
4 & 0.648817 & 4 & 0.660780 \\
5 & 0.687804 & 5 & 0.704639 \\
6 & 0.714731 & 6 & 0.725441 \\
\hline
\end{tabular}

(A)
(B)

\section{TABLA IV}

Correlaciones para cada sujeto en los experimentos $1 A$ y $1 B$ en soluciones $M D S$ tetradimensionales (Método Indscal).

\begin{tabular}{rrrr}
\hline \multicolumn{2}{c}{ Correlaciones } & \multicolumn{2}{c}{ Correlaciones } \\
\hline 1 & 0.838462 & 1 & 0.842206 \\
2 & 0.821529 & 2 & 0.592583 \\
3 & 0.813028 & 3 & 0.752433 \\
4 & 0.821074 & 4 & 0.790290 \\
5 & 0.708372 & 5 & 0.776522 \\
6 & 0.783872 & 6 & 0.827742 \\
7 & 0.874322 & 7 & 0.883242 \\
8 & 0.771259 & 8 & 0.776847 \\
9 & 0.792741 & 9 & 0.883203 \\
10 & 0.803005 & 10 & 0.801369 \\
11 & 0.780520 & 11 & 0.838407 \\
12 & 0.820629 & 12 & 0.850292 \\
13 & 0.831158 & 13 & 0.905364 \\
\hline
\end{tabular}

(A)

(B)

\section{Experimento 1B. Categorias de estructura difusa}

Una solución escalar de seis dimensiones parece explicar la mayor parte de la varianza. Observando la configuración del espacio de estímulo puede constatarse que las distintas alternativas no se distribuyen de forma aleatoria sino de acuerdo con los rasgos que el sujeto juzga relevantes en la comparación (cf. fig. 3. Representación de los estímulos. Experimento 1B. Categorías de estructura difusa). En la tabla IIIB se recoge la varianza que se explica en una solución de seis dimensiones. La primera dimensión de la configuración final viene representada por una propiedad configural: se trata de una relación de congruencia que el sujeto establece entre las dos figuras más externas. A su vez, el sujeto analiza estas figuras por separado reconociéndose su influencia en la segunda y cuarta dimensión -represen- 
tadas respectivamente por las figura inscrita y externa-. La figura inferior interna constituye la tercera dimensión y la figura superior interna la quinta. Por último, parece afectar a la varianza el valor de la figura del margen derecho cuya influencia se reconoce como una sexta dimensión, no siendo obvia la influencia de la figura que se presenta en el margen izquierdo. El sujeto opone entre sí las dos figuras más grandes y dependiendo de su con-

\section{FIGURA 3}

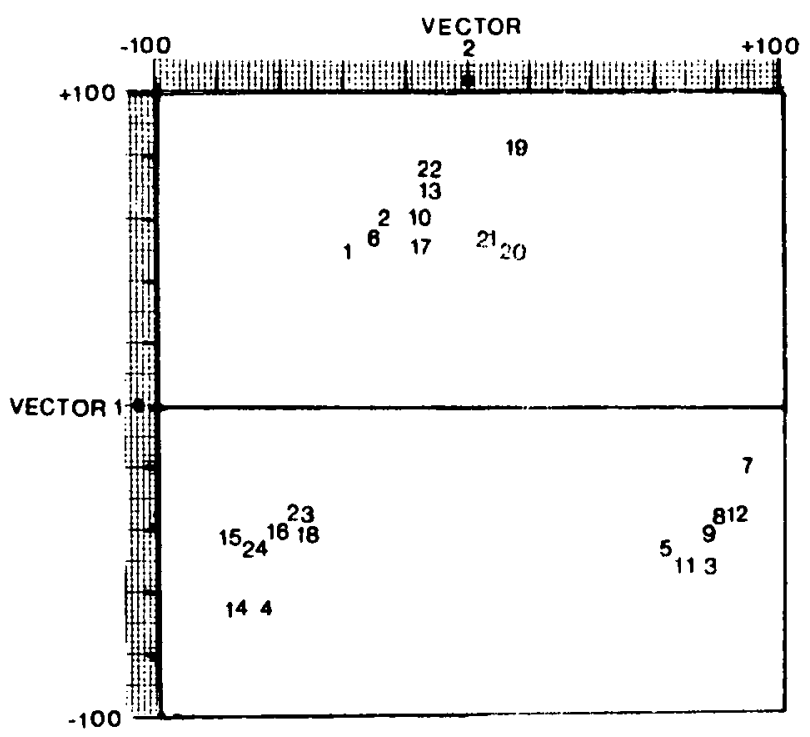

Representación de los estimulos. Exp. 1B. Categorias de estructura difusa.

gruencia decide la aplicación de otros criterios para evaluar la semejanza del par que se presenta. Los parámetros G, B, D, A, C, y F tienen en la clasificación. Las correlaciones que se obtienen entre las puntuaciones obtenidas en este análisis y los datos originales son sensiblemente distintas según los sujetos, poniendo de nuevo de manifiesto que cada uno de ellos evalúa de distinta manera este repertorio de estímulos (cf. tabla IVB).

\section{EXPERIMENTOS 2A-2B}

Los modelos de categorización que defienden la hipótesis distribucional del núcleo conceptual proponen reglas de transformación monotónica para explicar cómo procede el sujeto a clasificar una serie de estímulos cuando así se le propone en una tarea de clasificación. Depende de la distribución de sus propiedades que un estímulo se reconozca o no como ejemplar o miembro de un concepto. Sin embargo, el proceso de categorización no está directamente comprometido con el análisis perceptivo del estímulo, sino con su determinación significativa como miembro potencial de una clase de respuestas. El problema es decidir qué descripción de una serie de estímulos es apropiada dada una serie de respuestas alternativas a las que aquella serie conduce. Por hipótesis, el sistema cognitivo debe distribuir las alternativas de estímulo en términos de las alternativas de respuesta a que se asignan (cf. Farell, 1984; Nosofsky, 1985). Al ignorar cómo afecta al pro- 
ceso de categorización, qué alternativas de respuestas reconoce el sistema, los modelos de categorización ignoran la diferencia que existe entre el análisis del estímulo y su descripción como miembro de una clase de respuestas. En una tarea de clasificación el sistema puede reducir la información a la que es precisa para decidir, mediante una secuencia apropiada de tests, qué ejemplar es miembro de una categoría.

En esta segunda serie de experimentos se trata de decidir entre una hipótesis formal y una hipótesis distribucional del núcleo conceptual. Si como defienden los modelos probabilísticos y de ejemplar el proceso de categorización es de naturaleza monotónica, la descripción física del estímulo, es decir, su descripción estructural será suficiente para predecir cómo ha de comportarse el sistema cuando proceda a su clasificación. Los déficits significativos que se observen en el rendimiento de estos modelos predictivos mostrarán en qué medida es razonable la hipótesis que afirma el carácter no monotónico del proceso de categorización. La validez de esta hipótesis puede confirmarse reconstruyendo el proceso que lleva a los sujetos a decidir entre las distintas alternativas.

Con el fin de calcular las predicciones de los distintos modelos se calcularon las distancias entre los estímulos sometiendo los resultados obtenidos en los experimentos $1 \mathrm{~A}$ - categorías de estructura bien definida- y $1 \mathrm{~B}$ - categorías de estructura difusa- a un análisis de escalamiento multidimensional (cf. Kruskal y Wish, 1978). Las predicciones generales se obtuvieron promediando las puntuaciones de la matriz de pesos en cada dimensión.

El modelo de semejanza familiar - una descripción, en rigor, de la estructura del núcleo conceptual de una categoría - no utiliza estas distancias. por el contrario, se limita a calcular la semejanza de cada ejemplar con los de su categoría y los de la categoría alternativa según las propiedades que respectivamente las caracterizan (cf. Rosch y Mervis, 1975). Dado que el modelo de contexto es formalmente idéntico al modelo de densidad categorial no incluimos predicciones específicas de este último, menos preciso y general cuando se definen a priori las alternativas de estímulo y de respuesta (Fried y Holyoak, 1984).

El modelo de contexto supone que la latencia de respuesta es función de la evidencia que favorece la clasificación de un ejemplar como miembro de una cierta categoría. Esta evidencia se expresa formalmente como una proporción entre la semejanza ponderada de un ejemplar con los miembros de su categoría y la semejanza ponderada de este ejemplar con todos los ejemplares del contexto (cf. Medin y Schaffer, 1978). Con objeto de comparar el rendimiento de este modelo con otros del mismo tipo se calcularon las predicciones de los modelos de ejemplar promedio (cf. Hyman y Frost, 1975) y de ejemplar próximo (cf. Teed, 1972). El modelo de ejemplar promedio supone que la latencia de respuesta es función de la distancia media de un ejemplar a los miembros de su categoría menos la distancia media de este ejemplar a los de la categoría alternativa. El modelo de ejemplar próximo afirma que la latencia de respuesta es función de la distancia al más cercano de la categoría alternativa. Dado que en todas estas predicciones se utilizaron las distancias obtenidas a partir del método Indacal de escalamiento multidimensional, los parámetros de semejanza del modelo de contexto se estimaron de acuerdo con este método a partir de la varianza 
que explica cada una de las dimensiones en que se distribuyen los ejemplares.

Medin y Schaffer (1978) estiman los parámetros de semejanza de una serie de estímulos calculando los pesos que maximizan las correlaciones entre los resultados predichos por distintos modelos y los obtenidos en una tarea de clasificación. La identidad formal entre la regla de procedimiento del modelo de contexto y la regla de respuesta del modelo de Luce (1963) (cf. Nosofsky, 1984), nos permite definir estos parámetros recurriendo a las soluciones obtenidas en el análisis de escalamiento multidimensional. Al parámetro de semejanza entre un par de estímulos idénticos se le asignó el valor 10 , multiplicándose por este mismo valor la varianza normalizada explicada por cada dimensión.

El modelo de prototipo predice que la latencia de respuesta es función de la distancia de un ejemplar al prototipo de su categoría menos su distancia al de una categorpia alternativa (Posner y Keele, 1968). El prototipo de una categoria viene dado por las coordenadas del centroide en el espacio de estímulo proporcionado por el análisis de escalamiento multidimensional (cf. Martin y Caramazza, 1980).

En el contexto de una tarea de clasificación, el sistema cognitivo debe servirse de un sistema no monotónico de reglas para efectuar sus decisiones, reflejando la actuación de procesos asimétricos de inferencia que determinan qué rasgos resultan ser críticos para decidir la asignación de un ejemplar a una cierta categoría. Si se confirma este sistema no montónico de reglas de inferencia contradice la hipótesis que defiende que el proceso de categorización depende de una transformación monotónica que se efectúa a partir del análisis perceptivo del estímulo sobre las propiedades que resultan de este análisis. Parece razonable esperar que el sistema proceda, de acuerdo con esta hipótesis general, siguiendo: a) unas estrategias de procesamiento comunes a la hora de describir una serie de estímulos, tanto si responden a categorías de estructura bien definida, como si responden a categorías de estructura difusa; b) una heurística determinista para la asignación de una categoría - una alternativa de respuesta - a una alternativa de estímulo, regulándola en última instancia por los estados terminales a que conduce, y que c) esta heurística determinista deba regirse por algún sistema no monotónico de reglas de inferencia. Para confirmar estas hipótesis se sometió a un mismo grupo de sujetos a los experimentos que ahora se presentan. Tanto en el primer experimento como en el segundo, los sujetos debían aprender a clasificar los estímulos en términos de dos categorías de estructura bien definida - experimento $2 \mathrm{~A}$ - o en términos de dos categorías de estructura difusa -experimento $2 \mathrm{~b}$ -

\section{Método}

\section{Sujetos}

En estos experimentos participaron 22 estudiantes universitarios que se prestaron voluntariamente a colaborar en esta investigación: 12 en el experimento $2 \mathrm{~A}$-categorías de estructura bien definida-, y 10 en el experimento $2 \mathrm{~B}$ - categorías de estructura difusa-. Los mismos sujetos habian participado en los experimentos $1 \mathrm{~A}$ y $1 \mathrm{~B}$. 


\section{Diseño}

Los estímulos utilizados fueron idénticos a los que se presentaron en los dos experimentos previamente presentados. Las únicas variables que se manipularon son, por tanto, la estructura que definen las respectivas categorías y, por consiguiente, aquella que define cada uno de los ejemplares. En el experimento $2 \mathrm{~A}$, la asignación de una categoría puede llevarse a cabo mediante una regla conjuntiva que afecta únicamente a dos rasgos o propiedades de los ejemplares. En el experimento 2B, el proceso que permite asignar una categoría no es obvio: un ejemplar se parece más a los miembros de la categoría a que pertenece que a los de la alternativa. Las variables dependientes son la latencia de respuesta (TR), el número de errores (ER), y el último ensayo en que se comete un error durante el aprendizaje (UEE).

\section{Procedimiento}

El procedimiento es semejante en ambos experimentos. El sujeto debe, en ambas experiencias, aprender a clasificar los ejemplares en sus respectivas categorias. Los estímulos, fácilmente identificables, se presentaban aleatoriamente de modo que se redujera al mínimo el efecto de la secuencia en el proceso de aprendizaje. No se proporcionaba ningún tipo de información sobre la estructura de las categorías implicadas. Al término de la tarea, una vez concluido el aprendizaje de la clasificación y registradas las latencias de respuesta de otras tres últimas series correctas, el sujeto elaboraba un breve informe de cómo había aprendido a clasificar los ejemplares y de qué criterios se había servido para decidir sus respuestas. Los estímulos se presentaron mediante dos proyectores Kodak Carrousel 2050SAV controlados por un computador Hewlett Packard (HP 9825A) a través de un equipo multicanal (Scanner HP 3495A). El computador seleccionaba aleatoriamente el estímulo a presentar, controlaba la duración de su exposición y una vez que el sujeto proporcionaba su respuesta a través de un dispositivo que paraba un contador (Campden Instruments Timer counter 565), emitía a través de un generador de señales acústicas una señal que le indicaba al sujeto si su clasificación había sido o no correcta.

El sujeto iniciaba la prueba pulsando un botón. Tras un intervalo de 500 mseg aparecía en pantalla el primer estímulo durante un tiempo máximo de cuatro segundos. El sujeto debía responder durante este período de exposición. El estímulo desaparecía de la pantalla una vez el sujeto pulsaba el botón de respuesta $-\mathrm{A}$ o $\mathrm{B}-$ según creyera a qué categoría debía pertenecer el ejemplar. Al término de un ensayo recibía la señal que le indicaba si su respuesta había sido o no correcta. En el caso en que el sujeto no proporcionara su respuesta dentro del límite temporal establecido se contabilizaba como un error, y se procedía a la presentación del siguiente ensayo. Cada serie de ensayos se repetía un número limitado de veces, variable para cada sujeto, según necesitara éste hasta concluir su aprendizaje. Cada serie constaba de veinticuatro ensayos presentados en un orden aleatorio. Al término de una serie en la que el sujeto había cometido algún error de clasificación se presentaba la misma serie en un orden distinto, hasta un número máximo de treinta y cinco presentaciones. Si el sujeto llegaba a las veinte presentaciones de la serie sin haber aprendido como se clasifi- 
caban los ejemplares se le proporcionaba un descanso de dos horas para reanudar la tarea en una segunda sesión.

Después de aprender a clasificar correctemente los 24 estímulos de una serie, se le proporcionaban al sujeto tres nuevas series de ensayos sin feedback, durante las que se procedia a controlar el tiempo de reacción. A los sujetos se les instruía para que respondieran tan rápidamente como pudieran sin cometer errores. La presentación de cada serie llevaba aproximadamente cuatro minutos.

\section{Resultados y discusión}

Las tablas V y VIII recogen las predicciones de los distintos modelos de categorización cuando se presentan estímulos cuya clasificación responde a un sistema de categorias de estructura bien definida -experimento $2 \mathrm{~A}-$ (tabla V) o a un sistema de categorías de estructura difusa - experimento $2 \mathrm{~B}$ - (tabla VIII). En estas mismas tablas se incluyen las correlaciones que se obtienen entre estas predicciones y los promedios de las distintas variables dependientes para cada ejemplar. Las predicciones se efectuaron a partir de los análisis de escalamiento multidimensional llevados a cabo sobre los resultados obtenidos con los mismos sujetos en los experimentos $1 \mathrm{~A}$ y $1 \mathrm{~B}$.

\section{Experimento 2A. Categorias de estructura bien definida}

Como puede observarse en la tabla V, las correlaciones entre el índice número de rasgos característicos (N, PD1, PD2) y las distintas variables dependientes son elevadas, tanto en una como en otra categoría y mejor aún cuando no se pondera este predictor $(\mathrm{N})$. Las correlaciones entre las predicciones de los diversos modelos y las distintas variables dependientes son, por el contrario, desiguales.

Las correlaciones que se obtienen entre las predicciones de los modelos de ejemplar próximo, de prototipo y de contexto y las tasas medias de error (ER) son claramente inferiores en una categoría si se comparan con las que se obtienen en la otra. El modelo de contexto es el que mejor predice el comportamiento de esta variable, si se exceptúa el papel que puede cumplir satisfactoriamente el índice $\mathrm{N}$-número de rasgos característicos- cuando se emplea como predictor. Sometiendo a un análisis de varianza las tasas de error obtenidas, no observamos ningún efecto significativo de la variable de categoría, $\mathrm{F}(1,11)=0.76, \mathrm{p}>.05$; pero, si de la variable de ejemplar, $\mathrm{F}(11,121)=3.36, \mathrm{MSe}=1.32257, \mathrm{p}<.0005$, y de su interacción $\mathrm{F}(11,21)=8.65, \mathrm{MSe}=1.23143, \mathrm{p}<.0001$. Sin embargo, las medidas de esta variable dependiente muestran un aspecto terminal del proceso más que proporcionar una cierta información acerca de él. No es un tipo de medida que pueda obtenerse en tiempo real. Las bajas correlaciones que se obtienen en, al menos, una categoría confirman que existe una forma dominante de clasificación.

Las correlaciones que se obtienen entre las predicciones de los distintos modelos y los promedios de la variable último ensayo con error (UEE) no son equivalentes en ambas categorías. El modelo de ejemplar promedio es 
TABLA V. Predicciones y correlaciones. Exp. 2A, categorias de estructura bien definida.

\section{RASGOS DEFINITORIOS}

\begin{tabular}{|c|c|c|c|c|c|c|c|c|}
\hline \multirow{2}{*}{\multicolumn{2}{|c|}{$\begin{array}{l}\text { EJEMPLAR } \\
\text { CATEG. }\end{array}$}} & \multicolumn{3}{|c|}{ RASCOS } & \multirow{2}{*}{ PROMEDIO } & \multirow{2}{*}{ PROXIMO } & \multirow{2}{*}{ PROTOTIPO } & \multirow{2}{*}{ CONTEXTO } \\
\hline & & & & & & & & \\
\hline \multicolumn{2}{|c|}{1} & 4 & 52 & 608 & -.034 & -.236 & -.162 & .056 \\
\hline \multicolumn{2}{|c|}{2} & 3 & 51 & 584 & -.032 & -.245 & -.193 & .046 \\
\hline \multicolumn{2}{|c|}{3} & 3 & 48 & 572 & -.031 & -.213 & -.131 & .075 \\
\hline \multicolumn{2}{|c|}{4} & 2 & 47 & 548 & -.033 & -.234 & -.159 & .075 \\
\hline \multicolumn{2}{|c|}{5} & 3 & 48 & 568 & -.033 & -.224 & -.164 & .095 \\
\hline \multicolumn{2}{|c|}{6} & 2 & 47 & 544 & -.028 & -.152 & -.171 & .090 \\
\hline \multicolumn{2}{|c|}{7} & 3 & 51 & 584 & -.026 & -.175 & -.116 & .035 \\
\hline \multicolumn{2}{|c|}{8} & 2 & 50 & 560 & -.026 & -.232 & -.154 & .037 \\
\hline \multicolumn{2}{|c|}{9} & 2 & 47 & 548 & -.025 & -.177 & -.085 & .051 \\
\hline \multicolumn{2}{|c|}{10} & 1 & 46 & 524 & -.025 & -.211 & -.135 & .151 \\
\hline \multicolumn{2}{|c|}{11} & 2 & 47 & 544 & -.025 & -.219 & -.127 & .050 \\
\hline \multicolumn{2}{|c|}{12} & 1 & 46 & 520 & -.090 & -.174 & -.129 & .176 \\
\hline \multirow{9}{*}{ 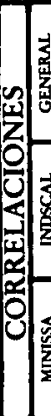 } & ER & -.75 & -.52 & -.70 & .73 & .40 & .40 & .77 \\
\hline & UEE & -.74 & -.50 & -.68 & .69 & .22 & .35 & .76 \\
\hline & TR & -.90 & -.76 & -.91 & .57 & .53 & .23 & .74 \\
\hline & ER & & & & -.16 & .03 & -.08 & .22 \\
\hline & UEE & & & & -.20 & .04 & -.11 & .16 \\
\hline & TR & & & & .11 & .20 & .15 & .35 \\
\hline & ER & & & & .58 & .00 & .59 & .30 \\
\hline & UEE & & & & .59 & .09 & .59 & .29 \\
\hline & TR & & & & .72 & .16 & .73 & .00 \\
\hline
\end{tabular}

RASGOS DEFINITORIOS

\begin{tabular}{|c|c|c|c|c|c|c|c|c|}
\hline \multicolumn{2}{|c|}{$\begin{array}{l}\text { JEMPLAR } \\
\text { CATEG. } \\
\end{array}$} & \multicolumn{3}{|c|}{ RASCOS } & PROMEDIO & PROXIMO & PROTOTTPO & CONTEXTO \\
\hline & 1 & 1 & 46 & 388 & .005 & -.182 & -.066 & .758 \\
\hline & 2 & 2 & 47 & 412 & .009 & -.172 & -.041 & .219 \\
\hline & 3 & 2 & 47 & 412 & .013 & -.197 & -.132 & .308 \\
\hline & 4 & 3 & 48 & 436 & .009 & -.180 & -.106 & .378 \\
\hline & 5 & 1 & 46 & 428 & .009 & -.180 & -.045 & .673 \\
\hline & 6 & 2 & 47 & 452 & .018 & -.218 & -.078 & .184 \\
\hline & 7 & 2 & 50 & 484 & .024 & -.288 & -.173 & .043 \\
\hline & 8 & 3 & 51 & 508 & .023 & -.267 & -.195 & .043 \\
\hline & 9 & 2 & 47 & 452 & .020 & -.300 & -.131 & .244 \\
\hline & 10 & 3 & 48 & 476 & .026 & -.310 & -.122 & .247 \\
\hline & 11 & 3 & 51 & 508 & .032 & -.363 & -.205 & .062 \\
\hline & 12 & 4 & 52 & 532 & .185 & -.356 & -.206 & .062 \\
\hline & ER & -.79 & -.68 & -.59 & -.36 & .53 & .68 & .57 \\
\hline & UEE & -.86 & -.77 & -.69 & -.44 & .63 & .75 & .85 \\
\hline 딕잉 & TR & -.83 & -.87 & -.73 & -.55 & .72 & .80 & .78 \\
\hline & ER & & & & -.17 & .27 & .24 & .33 \\
\hline & UEE & & & & -.18 & .29 & .25 & .31 \\
\hline & TR & & & & -.36 & .40 & .36 & .35 \\
\hline & ER & & & & -.42 & .30 & .51 & .65 \\
\hline & UEE & & & & -.51 & .41 & .59 & .66 \\
\hline & TR & & & & -.49 & .44 & .56 & .47 \\
\hline
\end{tabular}


el más irregular: el signo de la correlación se invierte al pasar de una a otra categoría. Los modelos de prototipo, de ejemplar próximo y de contexto, efectúan las mejores predicciones, pero son significativamente mejores en la categoría B, si se exceptúa el modelo de Medin y Schaffer (1978). Ningún modelo explica, no obstante, a qué se deben estos resultados, más aún si se tiene en cuenta que, en teoría, estos estímulos pueden ser exhaustivamente clasificados según una regla conjuntiva que todos y cada uno de los ejemplares satisface por igual. Amparado en este supuesto, el modelo clásico de categorización prediciría un comportamiento semejante en ambas categorías y con cada uno de sus ejemplares.

Para comprobar esta hipótesis se llevó a cabo un análisis de varianza sobre estos datos. En este análisis no se obtuvo, en efecto, ninguna diferencia significativa entre las categorías $\mathrm{F}(1,11)=1.90, \mathrm{MSe}=4.75852, \mathrm{p}>0.05$; pero si, en cambio, entre los ejemplares, $\mathrm{F}(11,21)=3.04$, $\mathrm{MSe}=2.83336$, $\mathrm{p}<.005$; lo que contradice al modelo clásico. También resulta ser significativa su interacción, $\mathrm{F}(11,121)=5.17, \mathrm{MSe}=3.81293, \mathrm{p}<.0001$. Si el sujeto decide clasificar los estímulos de acuerdo con un sistema de reglas lógicas, éstas no tienen la forma de una regla conjuntiva que se aplica a propiedades de estímulo no asimétricas. Los miembros de estas categorías, a pesar de los rasgos que comparten, no se tratan de la misma forma, incluso cuando estos rasgos bastarían por sí solos para su clasificación. Estos resultados ponen en cuestión la versión más primitiva del modelo clásico y parecen ajustarse mejor a una hipótesis distribucional del núcleo conceptual.

Al igual que se hiciera con las otras dos variables dependientes, se calcularon las latencias medias de respuesta a partir de las obtenidas por cada sujeto. La tabla $\mathrm{V}$ muestra las correlaciones que se obtienen entre las distintas predicciones y los promedios de tiempo de reacción obtenidos. El rendimiento de cada uno de los modelos varía dependiendo de la categoría. El modelo de ejemplar promedio vuelve a ser el más irregular: el signo de las correlaciones se invierte dependiendo de la categoría que se juzga. El modelo de ejemplar próximo predice las latencias de respuesta (TR) mejor que la tasa de errores (ER) y el último ensayo de error (UEE) y mejor tratándose de la categoría B que tratándose de la categoría A. Lo mismo ocurre con el modelo de prototipo que discrimina bien los miembros de la categoría B y mal, en cambio, los de la categoría A. De nuevo parece existir una categoría dominante a la hora de efectuar la clasificación. Aunque se observa una leve diferencia entre las categorías B y A en el modelo de contexto, éste es el que predice mejor el comportamiento de esta variable. Sin embargo, el mejor predictor de cómo se comporta esta variable es el índice número de rasgos característicos, tanto en su fomra simple como en su forma ponderada (N, PD1, PD2). Este predictor es la mejor expresión del modelo de semejanza familiar de Rosch y Mervis (1975): expresa la semejanza entre sí de los ejemplares, variable que determina, a juicio de estos autores, los tiempos de reacción que se observan.

Las correlaciones que se obtienen entre las predicciones de cada modelo y las latencias de respuesta son escasamente significativas. El rendimiento de estos modelos depende, casi exclusivamente, de cómo describen los estímulos, no de las estrategias que individualmente concibe cada sujeto para efectuar su clasificación (Sainz, 1985a) (cf. tabla V).

Para comprobar que los sujetos habían utilizado en sus clasificaciones di- 
ferentes estrategias se llevó a cabo un análisis de escalamiento multidimensional sobre las latencias de respuesta obtenidas. El método Indscal proporciona una correlación que expresa en qué medida se ajustan los datos originales de cada sujeto y las distancias calculadas por este método para cada uno de ellos. Escogiendo una solución de cuatro dimensiones explicamos el $78 \%$ de la varianza. Los coeficientes de correlación que se obtienen muestran que las estrategias de clasificación varían entre los sujetos (cf. tabla VI).

Cuando se someten las latencias de respuesta a un análisis de varianza se confirma que los sujetos no tratan los miembros de una categoría de la misma forma a pesar de que todos ellos comparten rasgos que bastarían para

\section{TABLA VI}

Correlaciones con los datos originales. Experimento $2 \mathrm{~A}$

\begin{tabular}{ccrl}
\hline Suj. & Correl. & Suj. & Correl. \\
\hline 1 & .370423 & 7 & .670749 \\
2 & .541804 & 8 & .681158 \\
3 & .377836 & 9 & .669222 \\
4 & .695336 & 10 & 561649 \\
5 & .603794 & 11 & .436700 \\
6 & .116410 & 12 & .562091 \\
\hline
\end{tabular}

TABLA VII

Correlaciones con los datos originales. Experimento $2 B$

\begin{tabular}{crrr}
\hline Suj. & Correl. & Suj. & \multicolumn{1}{c}{ Correl. } \\
\hline 1 & .457636 & 6 & .589075 \\
2 & .886983 & 7 & .141829 \\
3 & -.031722 & 8 & -.177604 \\
4 & .632309 & 9 & .459304 \\
5 & .527787 & 10 & .366098 \\
\hline
\end{tabular}

efectuar su clasificación. En efecto, la variable de ejemplar resulta ser significativa con una $\mathrm{F}(11,21)=6.89, \mathrm{MCe}=41394.48462, \mathrm{p}<.0001$; así como su interacción con la variable de categoría, $F(11,121)=10.88$, $\mathrm{MCe}=63013.88820, \mathrm{p}<.0001$. La variable de categoria, en cambio, no lo es con una $\mathrm{F}(1,11)=.84, \mathrm{MCe}=200259.19949, \mathrm{p}>.05$. EStos resultados no varían si se normalizan las latencias de respuesta: la variable de ejemplar obtiene una $\mathrm{F}(11,121)=9.80, \mathrm{MCe}=0.00419, \mathrm{p}<.0001$, $\mathrm{y}$ la variable de categoría una $\mathrm{F}(1,11)=.82, \mathrm{MCe}=.02572, \mathrm{p}>.05$, no significativa. Su interacción vuelve a ser significativa con una $F(11,121)=14.94$, $\mathrm{MCe}=.00672, \mathrm{p}<.001$. Los sujetos distinguen los estímulos entre sí más que limitarse a decidir su respuesta recurriendo a una regla conjuntiva.

Aunque todos los ejemplares satisfacen, en principio, la regla en función de la que se construyeron, su clasificación no depende exclusivamente de los rasgos que se derivan de su análisis perceptivo. El proceso de decisión no afecta sólo a la ejecución de una respuesta, sino al mismo proceso de elaboración de la información que la activa. El procesamiento depende del 
modo en que se asignen las alternativas de estímulo a las alternativas de respuesta que se proporcionaron (cf. Sáinz, 1985b, 1986). «El tipo de procesamiento depende del tipo de asignación, y de este modo refleja una propiedad del conjunto de las alternativas posibles de entre las cuales se extrae una cierta presentación» (Farell, 1984, p. 61). Una alternativa indica que las propiedades de los estímulos no son relevantes sino por relación a las reglas que las incluyen en un conjunto significativo del que se extrae. «Un estímulo único no puede tener significado real sin referencia a un conjunto de estímulos ya que los atributos que los definen no pueden especificarse sin conocer qué alternativas representan" (Garner, 1966, p. 11). Las propiedades tienen valor en cuanto disciernen las relaciones en que participan en el conjunto total de estímulos, o en otros términos, «en cuanto contraponen una presentación a todas las que, definidas por las mismas propiedades, son posibles» (Sáinz, 1985b, p. 240).

Un análisis más detallado de las diferencias que existen entre las distintas latencias medias de respuesta nos permite conocer las alternativas de estímulo que configuran el proceso de decisión. Al comparar entre sí las latencias de respuesta nos proveemos de una descripción del tipo de representaciones que de este dominio de estímulos forma el sujeto. La figura 4

Figura 4

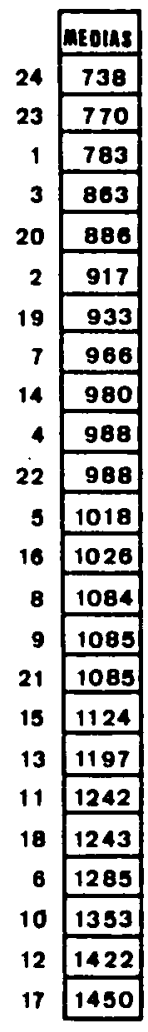

FIGURA 5

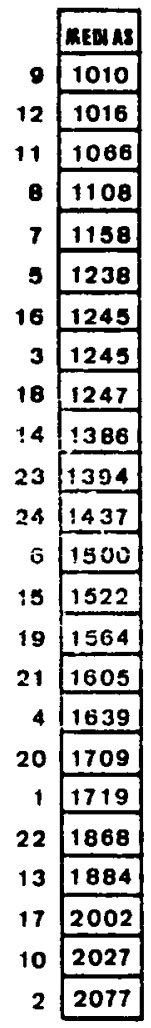

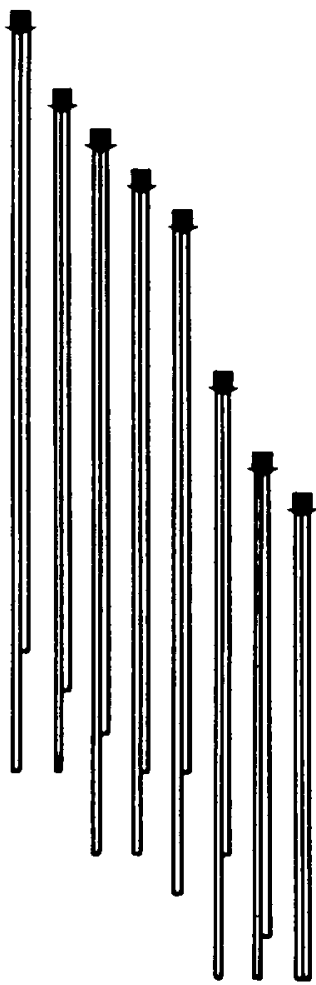

Diferencias significativas entre latencias de respuesta (prueba de Duncan). Exp. $2 B$. 
recoge las diferencias significativas que se obtienen al efectuar esta comparación. Contrastando las alternativas de estímulo con los datos que aporta cada sujeto en el informe que proporcionaba al término de su tarea, es posible reconstruir el proceso de decisión que explica nuestros resultados. Este proceso puede expresarse en términos de un árbol de decisión o un sistema de producción. Estos formalismos permiten describir la regla que determina la asignación de los ejemplares según las propiedades por las que pueden ser efectivamente asignados a una de las alternativas de respuesta (cf. fig. 6). Este árbol de decisión podría eventualmente complicarse o simplificarse según los sujetos. Sin embargo, al contrario de lo que predice el modelo clásico de categorización en su formulación más primitiva (cf. Smith y Medin, 1981), las propiedades que combina la regla de decisión no son simétricas. En su lugar, y de acuerdo con una formulación irrestricta de este modelo, estas propiedades no sirven sino para especificar el proceso de decisión.

\section{Experimento 2B. Categorias de estructura difusa}

Los promedios de las variables dependientes, tasa de errores (ER), último ensayo con error (UEE) y tiempos de reacción (TR) se correlacionan con las predicciones de los distintos modelos obteniendo las correlaciones que figuran en la tabla VIII.

En relación con las medias que se obtienen en las distintas variables dependientes, el modelo de ejemplar promedio es el que efectúa predicciones menos ajustadas. El mejor comportamiento del modelo de ejemplar próximo a la categoría $A$ se reduce sensiblemente cuando se trata de la categoría $\mathrm{B}$, al igual que ocurre con el modelo de contexto. Los modelos de contexto, de prototipo y de semejanza familiar efectúan predicciones más acordes con los resultados que se obtienen en la categoría $B$ que con los que se obtienen en la categoría A. Las correlaciones que se obtienen, sin embargo, a partir de las predicciones del modelo de prototipo no difieren significativamente en ambas categorías. De acuerdo con sus respectivas predicciones todos los modelos muestran, en suma, que existe una categoria dominante a partir de la cual se clasifican todos los ejemplares.

A fin de profundizar en el análisis de este efecto se llevaron a cabo una serie de análisis de varianza sobre las distintas medidas de las variables dependientes. El análisis de las tasas medias de error (ER) no arroja ningún efecto significativo de la variable de categoría, $F(1,9)=.03$, $\mathrm{MCe}=10.21713, \mathrm{p}>.05$; pero sí, en cambio, de la variable de ejemplar con una $\mathrm{F}(11,99)=4.90, \mathrm{MCe}=6.98952, \mathrm{p}<.0001$, y de su interacción $\mathrm{F}(11,99)=4.90, \mathrm{MCe}=12.42319, \mathrm{p}<.05$. Los sujetos buscan la mejor descripción de un dominio de estímulos, cuando proceden a su clasificación, de acuerdo con las alternativas de respuesta que se les proporcionan. El análisis llevado a cabo sobre la variable Ultimo Ensayo con Error (UEE) arroja resultados equivalentes. En efecto, no es significativa la variable de categoría, $\mathrm{F}(1,9)=.98, \mathrm{MCe}=48.63935, \mathrm{p}>.05$; pero sí es significativa, en cambio, la variable de ejemplar $\mathrm{F}(11,99)=3.10, \mathrm{MCe}=26.58851$, $\mathrm{p}<.01$, y su interacción con la variable de categoría obteniendo una $\mathrm{F}(11,99)=5.05, \mathrm{MCe}=34.36157, \mathrm{p}<.0001$. Como medidas indirectas del 
TABLA VIII. Predicciones y correlaciones. Exp. $2 B$, categorias de estructura difusa.

\begin{tabular}{|c|c|c|c|c|c|c|}
\hline \multicolumn{6}{|c|}{ RASGOS DE SEMEJANZA } & \multirow{2}{*}{$\begin{array}{c}\text { CAT.A } \\
\text { CONTEXTo }\end{array}$} \\
\hline \multicolumn{2}{|c|}{$\begin{array}{c}\text { EJEMPLAR } \\
\text { CATEGORIA } \\
\end{array}$} & $\begin{array}{l}\text { SEMEJANZA } \\
\text { FAMILAR }\end{array}$ & PROMEDIO & PROXMMO & PROTOTPO & \\
\hline & 1 & 41 & .003 & .143 & .044 & .316 \\
\hline & 2 & 43 & .012 & .026 & .009 & .242 \\
\hline & 3 & 41 & .000 & -.241 & -.150 & .171 \\
\hline & 4 & 35 & .002 & .299 & .120 & .492 \\
\hline & 5 & 47 & .004 & -.288 & -.171 & .142 \\
\hline & 6 & 37 & .007 & .217 & .111 & .573 \\
\hline & 7 & 37 & .029 & -.291 & -.181 & .140 \\
\hline & 8 & 41 & .027 & -.283 & -.175 & .223 \\
\hline & 9 & 43 & .013 & -.301 & -.153 & .210 \\
\hline & 10 & 39 & .015 & -.015 & -.007 & .247 \\
\hline & 11 & 43 & .028 & -.315 & -.183 & .276 \\
\hline & 12 & 43 & .065 & -.316 & -.167 & .244 \\
\hline & $\mathrm{TP}$ & -.62 & .10 & -.96 & -.95 & -.73 \\
\hline $\overrightarrow{\mathbf{s}}$ & ER & .61 & -.10 & .93 & .92 & .62 \\
\hline 宸 & UEE & .52 & -.13 & .83 & .81 & .47 \\
\hline 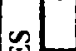 & TR & 28 & -.12 & .74 & .75 & .32 \\
\hline$z$ & $\mathrm{TP}$ & & -.68 & -.68 & -.68 & -.49 \\
\hline 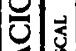 & $\mathrm{ER}$ & & .57 & .57 & .58 & .39 \\
\hline 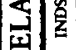 & $\overline{\mathrm{UEE}}$ & & .44 & .43 & .46 & .25 \\
\hline 五 & TR & & .38 & .42 & .43 & .17 \\
\hline 8 & $\mathrm{TP}$ & & -.95 & -.95 & -.95 & -.52 \\
\hline 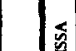 & ER & & .92 & .92 & .91 & .56 \\
\hline$\frac{2}{2}$ & UEE & & .81 & .81 & .81 & $: 53$ \\
\hline & $\operatorname{TR}$ & & .69 & .70 & .69 & .59 \\
\hline
\end{tabular}

\begin{tabular}{|c|c|c|c|c|c|c|}
\hline \multicolumn{6}{|c|}{ RASGOS DE SEMEJANZA } & \multirow{2}{*}{$\frac{\text { CAT. }}{\text { B }}$} \\
\hline \multicolumn{2}{|c|}{$\begin{array}{l}\text { EJEMPLAR } \\
\text { CÁTEGORIA } \\
\end{array}$} & $\begin{array}{c}\text { SEMEJANZA } \\
\text { FAMILIAR } \\
\end{array}$ & PROMEDIO & PROXIMO & PROTOTIPO & \\
\hline & 1 & 35 & .020 & -.006 & -.088 & .339 \\
\hline & 2 & 41 & .024 & .018 & -.112 & .189 \\
\hline & 3 & 39 & .024 & -.017 & -.152 & 195 \\
\hline & 4 & 43 & .012 & .025 & -.141 & .117 \\
\hline & 5 & 39 & .011 & .057 & .007 & .539 \\
\hline & 6 & 47 & .014 & -.057 & -.116 & .128 \\
\hline & 7 & 43 & .009 & -.050 & -.035 & .310 \\
\hline & 8 & 43 & .009 & -012 & -.040 & .342 \\
\hline & 9 & 39 & .005 & -.125 & -.040 & .272 \\
\hline & 10 & 39 & .010 & .141 & .013 & .572 \\
\hline & 11 & 45 & .020 & -.026 & -.116 & .170 \\
\hline & 12 & 41 & .115 & -.045 & -.104 & .289 \\
\hline & $\mathrm{TP}$ & -.82 & .29 & -.42 & -.45 & -.63 \\
\hline 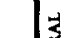 & ER & .79 & -.25 & .30 & .71 & .80 \\
\hline 恖 & UEE & .75 & -.14 & .05 & 69 & .72 \\
\hline 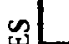 & TR & .71 & -.22 & .48 & .78 & .91 \\
\hline z & TP & & .15 & -.16 & .13 & -.20 \\
\hline 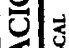 & ER & & -.32 & .07 & .35 & .33 \\
\hline 고로 & UEE & & -.21 & .00 & .25 & .21 \\
\hline 空 & TR & & -.23 & .15 & .31 & .36 \\
\hline u & $\mathrm{TP}$ & & .57 & -.12 &. .57 & -.26 \\
\hline 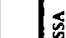 & ER & & -.84 & .16 & .85 & .31 \\
\hline 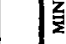 & UEE & & -.73 & .02 & .80 & .52 \\
\hline & TR & & -.84 & .18 & .82 & .38 \\
\hline
\end{tabular}


FIGURA 6

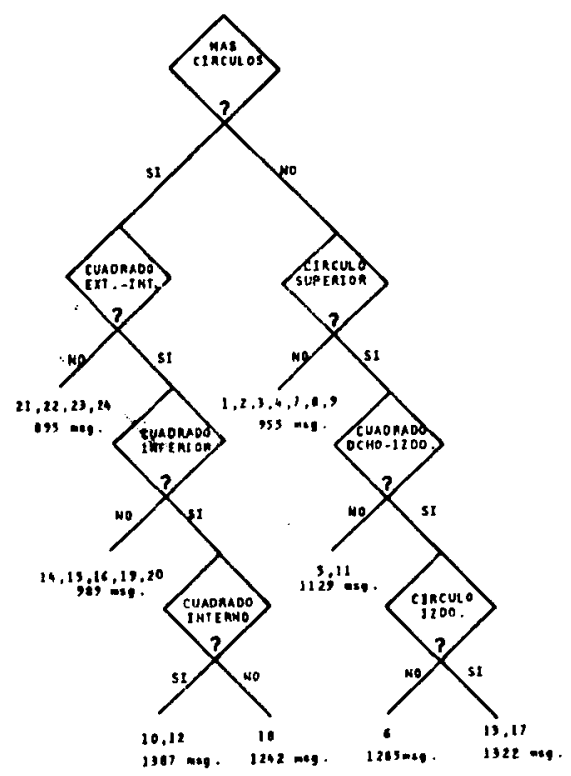

Arbol de decisión. Exp. $2 A$.
FIGURA 7

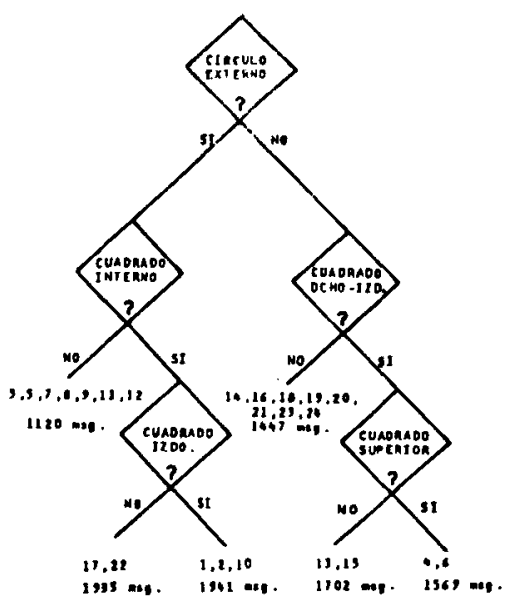

Arbol de decisión. Exp. $2 B$.

proceso de aprendizaje, el análisis de estas variables confirma que los distintos ejemplares no se aprenden con la misma facilidad. Este resultado debe atribuirse al análisis de la estructura del estímulo en relación con la alternativa de respuesta a que se asigna o representa. Que el sujeto busca una representación unitaria se deduce del hecho de que los errores no se distribuyan de acuerdo con la variable de categoría.

El análisis de varianza llevado a cabo sobre las latencias medias de respuesta confirma que los estímulos en esta condición experimental varían significativamente entre sí. Las diferencias que no se observan en el experimento $2 \mathrm{~A}$, sí se observan, en cambio, en éste. En efecto, las variables de categoría y de ejemplar resultan ser significativas con una $F(1,9)=7.14$, $\mathrm{MCe}=252416.32824, \mathrm{p}<.05$, y una $\mathrm{F}(11,99)=8.36$, $\mathrm{MCe}=131997.79356, \mathrm{p}<.0001$ respectivamente. También es significativa su interacción, $\mathrm{F}(11,99)=4.81, \mathrm{MCe}=211625.04945, \mathrm{p}<.0001$. Estos resultados no varían si el análisis de varianza se lleva a cabo sobre las latencias de respuesta normalizadas: las variables de categoría, $F(1,9)=9.52$, $\mathrm{MCe}=.03084, \mathrm{p}<.05$, y de ejemplar, $\mathrm{F}(11,99)=9.31, \mathrm{MCe}=.00959$, $\mathrm{p}<.0001$ son significativas. También es significatica su interacción, $\mathrm{F}(11,99)=6.81, \mathrm{MCe}=.01479, \mathrm{p}<.0001$. A pesar de que los sujetos intentan formar durante el aprendizaje una descripción unitaria del material que se somete a clasificación, sólo atienden a aquellas propiedades que resultan ser críticas para efectuar su clasificación, es decir, sólo a aquellas que permiten discernir entre las dos alternativas de respuesta. Estas propiedades definen las alternativas de estímulo en que se decide cómo se asignan a una u otra categoría cada ejemplar. La asignación de un ejemplar depende de la aplicación de una cierta secuencia de tests que define cómo se describe el dominio que se somete a clasificación. 
Para comprobar que los sujetos habían utilizado diferentes estrategias se llevó a cabo un análisis Indscal de escalamiento multidimensional sobre las latencias de respuesta obtenidas. Adoptando una solución de seis dimensiones puede explicarse el $82 \%$ de la varianza. Los coeficientes de correlación que se obtienen entre los datos originales y las puntuaciones calculadas para cada sujeto varían considerablemente como puede observase en la tabla VII.

Contrastando las latencias medias de respuesta es posible reconocer las distintas alternativas de estímulo en función de las que se configura el proceso de decisión (fig. 5) recoge las diferencias significativas que se obtienen al efectuar esta comparación. Estas diferencias pueden expresarse en términos de un árbol de decisión, que especifica cómo proceden los sujetos cuando clasifican los distintos ejemplares (cf. fig. 7). Según manifestaban los mismos sujetos al término de esta tarea, más que aprender qué conjunto de rasgos resultaba ser crítico para llevar a cabo la clasificación de los ejemplares, aprendian qué rasgos permitian decidir su asignación a una de las dos alternativas de respuesta. Cada una de las alternativas de estímulo distribuye el dominio en términos de una clase cuya identidad se define respecto de su complementaria. Un rasgo sólo es relevante si permite decidir entre las alternativas de respuesta. Como ocurriera en el experimento $2 \mathrm{~A}$, los sujetos construyen reglas que permiten la asignación estricta de un ejemplar supuesto que pertenece a un dominio definido por el conjunto de respuestas que le está asociado.

\section{DISCUSION GENERAL}

El primer experimento permite predecir, de acuerdo con los criterios de los distintos modelos de categorización, cómo debe comportarse el sistema cuando clasifica una serie de estímulos en términos de un sistema de categorías difusas o bien definidas. El rendimiento empírico de estas predicciones confirma, de acuerdo con Nosofsky (1985) que una métrica euclidiana es, en principio, adecuada para describir un dominio de estímulos de propiedades separables del que posteriormente se solicita su clasificación. Las descripciones que en una tarea de comparación efectúan los sujetos no son lineales. Sometiendo a un análisis de escalamiento multidimensional las puntuaciones de semejanza que se obtienen en esta tarea es posible reconocer cómo se distribuyen los distintos ejemplares de acuerdo con las alternativas de respuesta que se proporcionan.

Las predicciones de los distintos modelos de categorización no se ajustan satisfactoriamente a los resultados que se obtienen en el segundo experimento. Los modelos tienden a comportarse tanto mejor cuanto más se aproximan las demandas de la tarea a la descripción del conjunto de estímulos que se somete a clasificación. Esta descripción procede, en nuestro caso, de la comparación entre sí de los distintos estímulos y del análisis de escalamieto multidimensional que a este fin se lleva a cabo sobre los resultados obtenidos. Como señala Nosofsky (1984) la clasificación de un dominio, de acuerdo con una serie de alternativas de respuesta es, en todos y cada uno de los modelos de categorización propuestos, el resultado de una transformación lineal que se efectúa a partir de la mera descripción de 
sus propiedades. Dado que el sujeto no se sirve de estas propiedades, sino que las utiliza de forma contingente para decidir entre las distintas alternativas, estos modelos lineales no explican el proceso de categorización. Su naturaleza no lineal se confirma, en primer término, en el distinto tratamiento que cada sujeto hace de los ejemplares, de acuerdo con las categorías de que disponen, cuando procede a su clasificación.

$\mathrm{El}$ análisis de los resultados obtenidos en los experimentos $2 \mathrm{~A}$ y $2 \mathrm{~B}$ confirma que los sujetos utilizan las mismas categorías independientemente de la estructura interna de las categorias comprometidas. Estas estrategias de procesamiento deterministas, comunes a categorias de estructura difusa y a categorías de estructura bien definida, sólo pueden explicarse de acuerdo con los postulados teóricos del modelo clásico, el único que es explícito al defender la naturaleza formal del núcleo conceptual. De acuerdo con una heurística determinista, en efecto, un ejemplar se reconoce miembro de un concepto si satisface ciertas propiedades críticas. Sin embargo, frente al modelo clásico, la regla que especifica cómo se asignan los ejemplares a cada una de las categorías de respuesta no afecta por igual a todas las propiedades en que se describe el dominio que representan. Una propiedad es crítica si permite discernir entre las alternativas de respuesta (cf. McDaniel, 1984) de acuerdo con la regla de la que es criterio. Por la estructura asimétrica de sus propiedades, una regla puede representarse implícitamente por el orden en que éstas se activan cuando el sujeto identifica qué ejemplares son miembros de una cierta categoría.

Un modelo lineal de categorización supone que la mera descripción de un estímulo basta para decidir su clasificación en términos de las alternativas de respuesta o categorías que se proporcionan. Nuestros datos, sin embargo, no se ajustan a las predicciones de los modelos lineales de categorización propuestos hasta el momento. Los sujetos clasifican las distintas alternativas de estímulo de acuerdo con un sistema no monotónico de reglas de inferencia que permite decidir a qué categoría se asigna cada uno de los ejemplares. Los criterios en base a los cuales se decide la asignación de un ejemplar a una de las categorías de respuesta distribuyen los estímulos en clases complementarias, un tipo de transformación no lineal que resulta determinante en el proceso de categorización. De este modo, cada criterio especifica una producción que representa a todos los estímulos de los que es alternativa en una cierta etapa del proceso de decisión. Aquellos ejemplares para los que un cierto criterio basta, pueden clasificarse sin que sea necesaria la aplicación exhaustiva de la regla. Los modelos lineales hasta ahora propuestos explican el proceso de categorización sólo en el caso límite en que la regla de decisión reconoce las mismas alternativas de estímulo que se hallan implícitas en la mera descripción de los estímulos que se presentan. Nuevas experiencias permitirán profundizar y matizar este análisis.

\section{Referencias}

Aiken, L. S.; Fenker, R. M., Evans, R., y SELbY, H. (1974). Class structure in the biasing of perceived pattern similarity. Joumal of Experimental Psychology, 103 (3), 489-501.

ANDERSEN, E. S. (1975). Cups and glasses: Learning that boundaries are vague. Journal of Child Language, 2, 79-103.

Anderson, J. A., y Silverstein, J. W. (1978). Reply to Grossberg. Psychological Review, $85,597-603$. 
ANDERSON, J. A.; SILVERSTEIN, J. W.; RITZ, S. A., y JonES, R. S. (1977). Distinctive features categorical perception, and probability learning: Some applications of a neural model. Psychological Review, 84, 413-451.

ARMSTRONG, S. L.; Gleitman, L. R., y Gleitman, H. (1983). What some concepts might not be. Cognition, 13, 263-308.

BROOKS, L. R. (1978). Nonanalytic concept formation and memory for instances. En E. Rosch y B. Lloyd (eds.): Cognition and categorization (pp. 169-211. Hillsdale: Lawrence Erlbaum Associates.

Buffart, H.; LeEUWENBERG, E., y Restle, F. (1981). Coding theory of visual pattern completion. JEP: Human Perception and Performance, 7 (2), 241-274.

CHAFFIN, R. (1981). Context effects in the categorization task. Journal of General Psycho$\log y, 104(2), 293-302$.

CLANCEY, W. J. (1985). Heuristic classification. Artificial Intelligence, 27, 289-350.

DUNN, J. C. (1983). Spatial metrics of integral and separable dimensions. JEP: Human Perception and Performance, 9 (2), 242-257.

FalmaGnE, J. C. (1985). Elements of psychophysical theory. Nueva York: Oxford University Press.

FARELL, B. (1985). Attention in the processing of complex visual displays: Detecting features and their combinations. JEP: Human Perception and Performance, 10 (1), 40-64.

Elannagan, M.; FRIED, L. S., y HolyoaK, K. J. (1981). Perceptual category learning and distributional structure. Psychonomic Society 22nd Meeting: Filadelfia.

FOARD, C. F., y KEMLER NELSON, D. G. (1984). Holistic and analytic modes of processing: The multiple determinants of perceptual analysis. Joumal of Experimental Pyschology: General, 113 (1), 94-111.

FODOR, J. A. (1983). The modularity of mind. Cambridge, Mass.: MIT Press.

FRIED, L. S., y HOLYOAK, K. J. (1984). Induction of category distribution: A framework for classification learning. JEP: Learning,Memory and Cognition, 10 (2), 234-257.

HAMPTON, J. A. (1979). Polymorphous concepts in semantic memory. Journal of Verbal Learning and Verbal Behavior, 18, 441-461.

HAMPTON, J. A. (1982). A demostration of intrasitivity in natural categories. Cognition, 12 (2), 151-164.

HiNTON, G. E. (1981). Implementing semantic networks in parallel hardware. En G. E. Hinton y J. A. Anderson (comps.): Parallel models of associative memory, 161-187, Hillsdale: Erlbaum.

HYMAN, R., y Frost, N. H. (1975). Gradients and schema in pattern recognition. En P. M. A. Rabbit y S. Dornic (comps.): Attention and performance. Londres: Academic Press.

KNAPP, A. G., y ANDERSON, J. A. (1984). Theory of categorization based on distributed memory storage. JEP: Learning, Memory and Cognition, 10 (4), 616-637.

KRUSKAL, J. B., y WISH, M. (1978). Multidimensional scaling. Londres: Sage.

LABOV, W. (1973). The boundaries of words and their meanings. En C. J. N. Bailey y R. W. Shuy (comps.): New ways of analyzing variation in english, vol. Washington: Georgetown University Press.

LAKOFF, G. (1973). Hedges: A study in menaing criteria and the logic of fuzzi concepts. Journal of Philosophical Logic, 2, 458-508.

LuCE, R. D. (1963). Detection and recognition. En R. D. Luce,. R. R. Bush y E. Galanter (comps.): Handbook of Mathematical Psychology, 103-189. Nueva York: Wiley.

MALT, B. C., y SMITH, E. E. (1984). Correlated properties in natural categories. Journal of Verbal Learning and Verbal Behavior, 23, 250-269.

MARCEL, A. (1983a). Conscious and unconscious perception: Experiment on visual masking and word recognition. Cognitive Psychology, 15, 197-237.

MARCEL, A. (1983b). Conscious and unconscious perception: An approach to the relations between phenomenal experience and perceptual processes. Cognitive Psychology, 15, 238-300.

MARKMAN, E. (1984). The acquisition and hierarchical organization of categories by children. En C. Sophian (1984). Hilsdale: Lawrence Erlbaun Associates.

MARTIN, R. C., y Caramazza, A. (1980). Classification in well-define and ill-defined categories: Evidence for common processing strategies. Journal of Experimental Psychology: General, 109, 320-353.

MASSARO, D. W., y HARY, J. M. (1984). Categorical results, categorical perception and hindsight. Perception and Psychophysics, 35 (6), 586-588.

MCClelland, J. L., y RumelhaRT, D. E. (1985). Distributed memory and the representation of general and specific information. Journal of Experimental Psychology: General, $114(2), 159-188$.

MCCloskey, M., y Glucksberg, S. (1979). Decision processes in verifying class inclusion statements: Implication for models of semantic memory. Cognitive Psychology, 11, 1-37.

MCDANIEL, M. A. (1984). The role of elaborative processes and schema processes in story memory. Memory and Cognition, 12, 46-51.

MCDERmOTT, D. (1982). Nonmonotonic logic II. Nonmonotonic modal theories. J. $A C M$, 29 (1), 33-57. 
MCDermotT, D., y DOYLe, J. (1980). Non-monotonic logic I. Artificial Intelligence, 13 (1, 2), 41-72.

Medin, D. L., y SChaffer, M. (1978). Context theory of classification learning. Psychological Review, 85 (3), 207-238.

MEDIN, D. L. (1983). Structural principles in categorization. En T. J. Tighe y B. Sheep (comps.): Interactions: perception, cognition and development. Hillsdale: Lawrence Erlbaum Associates.

Medin, D. L.; Alton, M. W., y MuRPhy, T. D. (1984). Given versus induced category representations: Use of prototype and exemplar information in classification. JEP: Learning, Memory and Cognition, 10 (3), 333-352.

MERVIS, C. B. (1980). Category structure and development of categorization. En R. Spiro, B. C. Bruce y W. F. Brewer (comps.): Theoretical issues in reading comprehension. Hilldale: Lawrence Erlbaum Associates.

MERvis, C. B., y ROSCH, E. (1981). Categorization of natural objects. Annual Review, 32, 89-115.

MOORE, R. C. (1985). Semantical considerations on nonmonotonic logic. Artificial Intelligence, 25, 75-94.

NEUMANN, P. G. (1977). Visual prototype formation with discontinuous representation for dimensions of varibility. Memory and Cognition, 5 (2), 187-197.

NEWELL, A. (1982). The knowledge level. Artificial Intelligence, 18, 87-127.

NOSOFSKY, R. M. (1984). Choice, similarity, and the context theory of classification. JEP: Learning, Memory and Cognition, 10 (1), 104-114.

NOSOFSKY, R. M. (1985). Overal similarity and the identification of separable-dimension stimuli: A choice model analysis. Perception and Psychophysics, 38 (5), 415-432.

ODEN, G. (1977). Fuzziness in semantic memory: Choosing exemplars of subjetive categories. Memory and Cognition, 5 (2), 198-204.

OSHERSON, D. N., y SMITH, E. E. (1981). On the adequacy of prototype theory as a theory of concepts. Cognition, 9(1), 35-58.

Osherson, D. N., y SMITH, E. E. (1982). Gradedness and conceptual combination. Cognition, 12, 299-318.

Peterson, M. A., y HochBerg, J. (1983). Opposed-set measurement procedure: A quantitative analysis of the role of local cues and intention in form perception. JEP: Human Perception and Performance, 9 (2), 183-193.

PITZ, G. F., y LEUNG, L. S. (1977). Categorization and decision rules: A comparison of the likelyhood ratio y prototype models. Perceptual and Motos Skills, 45 (2), 539-549.

POSNER, M. E., y KEELE, S. W. (1968). On the genesis of abstract ideas. Journal of Experimental Psychology, 77 (3, PT.1), 353-363.

PyLYSHYN, Z. W. (1984). Computation and cognition. Cambridge, Mass: The MIT Press.

ReBER, A. S., y ALLEN, R. (1978). Analogical and abstraction strategies in synthetic grammar learning: A funcionalist interpretation. Cognition, 6, 189-221.

REED, S. K. (1972). Pattern recognition and categorization. Cognitive Psychology, 3 (3), 382-407.

REED, S. K., y FRIEDMAN, M. P. (1973). Perceptual vs. conceptual categorization. Memory and Cognition, 1 (2), 157-163.

RichaRdsON, K., y BHAVNANI, K. K. (1984). How a concept is formed: Prototype or contingency abstraction? British lournal of Psychology, 75, 507-519.

Rosch, E. (1978). Principles of categorization. En E. Rosch y B. Lloyd (comps.): Cognition and categorization (pp. 28-46). Hillsdale: Lawrence Erlbaum Associates.

ROSCH, E., y MERVIS, C. B. (1975). Family resemblances: Studies in the internal structure of categories. Cognitive Psychology, 7, 573-605.

ROTH, E. M., y MERvIS, C. B. (1983). Fuzzy set theory and class inclusion relations in semantic categories. Journal of Verbal Learning and Verbal Behavior, 22, 509-525.

SÁINZ, J. (1985a). Hacia un nuevo modelo de categorización. En J. Mayor (comp.): Actividad Humana y Procesos Cognitivos. Madrid: Alhambra (1985).

SÁINZ, J. (1985b). Factores de estímulo y de respuesta en el procesamiento de información: I. Asimetrias estructurales de procesamiento. Informes de Psicología, 223-248.

SÁINZ, J. (1986). Factores de estímulo y de respuesta en el procesamiento de información: II. Asimetrías de asignación y modelos de procesamiento. Informes de Psicología (en prensa).

SÁINZ, J., y GONZALEZ-MARQUES, J. (1986). Esquemas y guiones. Madrid: Alhambra.

SMITH, E. E., y MEDIN, D. (1981). Categories and concepts. Cambridge Mass: Harvard University Press.

SMITH, E. E., y OSHERSON, D. N. (1984). Conceptual combination with prototype concepts. Cognitive Science, 8, 337-361.

SUPPES, P. (1962). Models of data. En E. Nagel, P. Supes y A. Tarski (comps.): Logic, methodology and philosophy of science. Stanford: Stanford University Press. 